\title{
A new characterization of bivariate copulas
}

\author{
Fabrizio Durante and Piotr Jaworski* \\ Department of Knowledge-Based Mathematical Systems \\ Johannes Kepler University, A-4040 Linz, Austria \\ e-mail: fabrizio.durante@jku.at \\ Institute of Mathematics, Warsaw University \\ Banacha 2, 02-097 Warszawa, Poland \\ e-mail: jwptxa@mimuw.edu.pl
}

Received: December 15, 2008; Revised: June 24, 2009

\begin{abstract}
A new characterization of bivariate copulas is given by using the notion of Dini derivatives. Several examples illustrate the usefulness of this result.

Keywords: Copula, 2-increasing property, Dini derivative.

AMS Subject Classification: 60E05, 62H20, 26A24.
\end{abstract}

\section{Introduction}

A (bivariate) copula is a distribution function on $\mathbb{I}^{2}:=[0,1]^{2}$ whose univariate margins are uniformly distributed. Specifically, $C: \mathbb{I}^{2} \rightarrow \mathbb{I}$ is a copula if it satisfies the following properties:

(C1) $C(x, 0)=C(0, x)=0$ for every $x \in \mathbb{I}$, i.e. $C$ is grounded,

(C2) $C(x, 1)=C(1, x)=x$ for every $x \in \mathbb{I}$,

(C3) $C$ is $2-$ increasing, that is, for every $x_{1}, y_{1}, x_{2}, y_{2} \in \mathbb{I}, x_{1} \leq x_{2}$ and $y_{1} \leq y_{2}$, it holds

$$
C\left(x_{1}, y_{1}\right)+C\left(x_{2}, y_{2}\right) \geq C\left(x_{1}, y_{2}\right)+C\left(x_{2}, y_{1}\right) .
$$

${ }^{*}$ Corresponding author. Tel. 4822-554-45-23, Fax. 4822-554-43-00 
Recently, copulas have received a great popularity due to the celebrated Sklar's Theorem, stating that every joint distribution function of a pair of continuous random variables can be represented by means of a suitable copula and its univariate marginals. Just to have an idea about copula theory and (some of) its applications, we refer to Schweizer and Sklar (2006); Joe (1997); Nelsen (2006); McNeil et al. (2005); Salvadori et al. (2007).

The growing importance of copulas for constructing statistical models has originated several methods for generating new copulas. The final goal of these investigations is to obtain more flexible families of bivariate distribution functions, having a variety of interesting properties like tail dependencies, asymmetries, wide range of association.

From an abstract viewpoint, generating new copulas consists of defining a function $C: \mathbb{I}^{2} \rightarrow$ II and proving that it satisfies the required properties (C1), (C2) and (C3). Now, while (C1) and $(\mathrm{C} 2)$ are usually trivial to check, the verification of (C3) could be, sometimes, a tricky task that might bring us "into the exciting land of copula-exotics" (compare with Embrechts (2009)). In this paper, we show how the 2 -increasing property (C3) can be characterized in a different way by using the notion of Dini derivatives (section 2). This characterization allows us to provide some new and old constructions of copulas in a simple way (section 3).

\section{Dini derivatives and 2-increasing property}

The concept of Dini derivative (or Dini derivate) generalizes the classical notion of the derivative of a real-valued function. Here we recall its basic properties. For more details, we refer to Łojasiewicz (1988).

Let $a, b \in \mathbb{R}, a<b$, and let $f:[a, b] \rightarrow \mathbb{R}$ be a continuous function. Let $x$ be a point in $[a, b)$. The limits

$$
\begin{aligned}
& D^{+} f(x)=\limsup _{h \rightarrow 0^{+}} \frac{f(x+h)-f(x)}{h}, \\
& D_{+} f(x)=\liminf _{h \rightarrow 0^{+}} \frac{f(x+h)-f(x)}{h} .
\end{aligned}
$$

are called, respectively, rightside upper and lower Dini derivatives of $f$ at $x$. Note that the rightside Dini derivatives take values in $[-\infty,+\infty]$. Given two continuous functions $f_{1}, f_{2}$, it is easy to show that:

$$
\begin{aligned}
& D_{+}\left(f_{1}+f_{2}\right)(x) \geq D_{+} f_{1}(x)+D_{+} f_{2}(x), \\
& D^{+}\left(f_{1}+f_{2}\right)(x) \leq D^{+} f_{1}(x)+D^{+} f_{2}(x), \\
& D^{+}\left(f_{1}-f_{2}\right)(x) \geq D^{+} f_{1}(x)-D^{+} f_{2}(x),
\end{aligned}
$$

for every point $x$ that admits finite Dini derivatives of $f_{1}$ and $f_{2}$. Moreover, the above inequalities become equalities when $f_{2}$ is differentiable. 
Obviously, if $f$ is increasing on $[a, b]$, then $D^{+} f(x)$ and $D_{+} f(x)$ are nonnegative for every $x \in[a, b)$. For our purposes, the converse of the previous implication will be crucial (see (Łojasiewicz, 1988, Theorem 7.4.14)).

Lemma 2.1. If $f$ is continuous on $[a, b], D^{+} f(x)>-\infty$ except for at most a countable subset in $[a, b]$, and $D^{+} f(x) \geq 0$ almost everywhere in $[a, b]$, then $f$ is increasing on $[a, b]$.

In the following, for every function $H:\left[x_{1}, x_{2}\right] \times\left[y_{1}, y_{2}\right] \rightarrow \mathbb{R}$, we denote by $H_{y}$ the horizontal section of $H$ at the point $y \in\left[y_{1}, y_{2}\right]$, defined by

$$
H_{y}:\left[x_{1}, x_{2}\right] \rightarrow \mathbb{R}, \quad H_{y}(x)=H(x, y) .
$$

The following lemma will be essential in the sequel.

Lemma 2.2. Let $H: \mathbb{I}^{2} \rightarrow \mathbb{R}$ be a continuous function such that $D_{+} H_{0}(x)$ and $D^{+} H_{1}(x)$ are finite for every $x \in \mathbb{I} \backslash \mathscr{Z}$, where $\mathscr{Z}$ is a countable subset of $\mathbb{I}$. The following statements are equivalent:

(1) $H$ is 2-increasing.

(2) For each $x \in \mathbb{I} \backslash \mathscr{Z}$, the following conditions hold:

(i) $D^{+} H_{y}(x)$ and $D_{+} H_{y}(x)$ are finite for every $y \in \mathbb{I}$;

(ii) $D_{+} H_{z}(x) \geq D_{+} H_{y}(x)$ and $D^{+} H_{z}(x) \geq D^{+} H_{y}(x)$ whenever $0 \leq y<z \leq 1$.

(3) For each $x \in \mathbb{I} \backslash \mathscr{Z}_{1}$, where $\mathscr{Z}_{1}$ is a countable subset of $\mathbb{I}$, the following conditions hold:

(i) $D^{+} H_{y}(x)$ is finite for each $y \in \mathbb{I}$;

(ii) $D^{+} H_{z}(x) \geq D^{+} H_{y}(x)$ whenever $0 \leq y<z \leq 1$.

Proof. We will prove $(1) \Rightarrow(2) \Rightarrow(3) \Rightarrow(1)$.

$(1) \Rightarrow(2)$ : Let $x$ be in $\mathbb{I} \backslash \mathscr{Z}$. Since $H$ is 2-increasing, it follows that, for every $y, z \in \mathbb{I}$ such that $y<z$ and for every $h \in[0,1-x]$, we have

$$
H_{y}(x+h)-H_{y}(x) \leq H_{z}(x+h)-H_{z}(x),
$$

and, as a consequence,

$$
D^{+} H_{y}(x)=\limsup _{h \rightarrow 0^{+}} \frac{H_{y}(x+h)-H_{y}(x)}{h} \leq \limsup _{h \rightarrow 0^{+}} \frac{H_{z}(x+h)-H_{z}(x)}{h}=D^{+} H_{z}(x) .
$$

In the same way, we obtain $D_{+} H_{y}(x) \leq D_{+} H_{z}(x)$. In particular, for every $y \in \mathbb{I}$, we have

$$
D_{+} H_{0}(x) \leq D_{+} H_{y}(x) \leq D^{+} H_{y}(x) \leq D^{+} H_{1}(x) .
$$

Thus, rightside upper and lower Dini derivatives of $H_{y}(x)$ are finite for every $y \in \mathbb{I}$ and for every $x \in \mathbb{I} \backslash \mathscr{Z}$. 
$(2) \Rightarrow(3)$ : trivial.

$(3) \Rightarrow(1)$ : Let $x_{0}, y, z$ be in $\mathbb{I}, z>y$. Let $f_{x_{0}}: \mathbb{I} \rightarrow \mathbb{R}$ be the function defined by

$$
f_{x_{0}}(x)=H_{z}(x)-H_{y}(x)-H_{z}\left(x_{0}\right)+H_{y}\left(x_{0}\right) .
$$

Since $f_{x_{0}}\left(x_{0}\right)=0$, in order to prove that $H$ is 2 -increasing, it suffices to show that, for arbitrary $y, z \in \mathbb{I}$ such that $z>y, f_{x_{0}}$ is increasing. Now, for every $x \in \mathbb{I} \backslash \mathscr{Z}$, we have:

$$
D^{+} f_{x_{0}}(x)=D^{+}\left(H_{z}(x)-H_{y}(x)\right) \geq D^{+} H_{z}(x)-D^{+} H_{y}(x)
$$

and, as a consequence of $(3)$ (part (ii)), $D^{+} f_{x_{0}}(x) \geq 0$ for $x \in \mathbb{I} \backslash\left(\mathscr{Z}_{1} \cup \mathscr{Z}\right)$. Since Lemma 2.1 it follows that $f_{x_{0}}$ is increasing on $\mathbb{I}$, and, hence, non-negative on $\left[x_{0}, 1\right]$.

Now, we can state our main result, that is an immediate consequence of the previous lemma.

Theorem 2.3. A function $C: \mathbb{I}^{2} \rightarrow \mathbb{I}$ is a copula if, and only if, $C$ satisfies (C1), (C2) and the following conditions:

(C3') $C$ is continuous;

(C4') there exists a countable set $\mathscr{Z} \subset \mathbb{I}$ such that, for every $x \in \mathbb{I} \backslash \mathscr{Z}$, the following conditions hold:

(i) $D^{+} C_{y}(x)$ is finite for every $y \in \mathbb{I}$,

(ii) $D^{+} C_{z}(x) \geq D^{+} C_{y}(x)$ whenever $0 \leq y<z \leq 1$.

From the previous characterization, it follows that, when we would like to prove that a function $C: \mathbb{I}^{2} \rightarrow \mathbb{I}$ is a copula we can replace the proof of the 2 -increasing property (C3) with the verification of $\left(\mathrm{C}^{\prime}\right)$ and $\left(\mathrm{C}^{\prime}\right)$. In particular, when $C: \mathbb{I}^{2} \rightarrow \mathbb{I}$ admits continuous first partial derivatives, we obtain the following well-known characterization.

Corollary 2.4. Let $C: \mathbb{I}^{2} \rightarrow \mathbb{I}$ be a function that admits continuous first partial derivatives. Then, $C$ is a copula if, and only if, $C$ satisfies (C1), (C2) and, for every $x \in \mathbb{I}$,

$$
y \mapsto \frac{\partial C(x, y)}{\partial x}
$$

is increasing on $\mathbb{I}$.

Here, we would like to connect the characterization of Theorem 2.3 with the notion of quasi-copula, a generalization of the concept of copula introduced by Alsina et al. (1993). We recall that $C: \mathbb{I}^{2} \rightarrow \mathbb{I}$ is a quasi-copula if it is increasing in each variable, 1-Lipschitz, i.e., for every $x_{1}, x_{2}, y_{1}, y_{2} \in \mathbb{I}$,

$$
\left|C\left(x_{1}, y_{1}\right)-C\left(x_{2}, y_{2}\right)\right| \leq\left|x_{1}-x_{2}\right|+\left|y_{1}-y_{2}\right|
$$


and satisfies (C2) (compare with Genest et al. (1999)). In particular, for a quasi-copula $C$ it follows that $0 \leq D^{+} C_{y}(x) \leq 1$ for every $x \in[0,1)$ and $y \in \mathbb{I}$. Thus, as a consequence of Theorem 2.3, the following characterization holds.

Corollary 2.5. Let $C$ be a quasi-copula. Then $C$ is a copula if, and only if, for every $x \in \mathbb{I} \backslash \mathscr{Z}$, where $\mathscr{Z}$ is a countable subset of $\mathbb{I}, D^{+} C_{z}(x) \geq D^{+} C_{y}(x)$ whenever $0 \leq y<z \leq 1$.

Example 2.6. Let $C: \mathbb{I}^{2} \rightarrow \mathbb{I}$ be given by $C(x, y)=x y+f(y) \sin (2 \pi x)$, where

$$
f: \mathbb{I} \rightarrow\left[0, \frac{1}{24}\right], \quad f(y)= \begin{cases}0, & 0 \leq y<1 / 4, \\ \frac{4 y-1}{24}, & \frac{1}{4} \leq y<\frac{1}{2}, \\ \frac{1-y}{12}, & \frac{1}{2} \leq y \leq 1 .\end{cases}
$$

As shown by Genest et al. (1999), $C$ is a quasi-copula, but not a copula. In fact, it is easy to show that

$$
D^{+} C_{1 / 2}\left(\frac{1}{2}\right)=\frac{6-\pi}{12}<\frac{1}{4}=D^{+} C_{1 / 4}\left(\frac{1}{2}\right),
$$

hence, from the continuity of $D^{+} C_{y}(x)$ with respect to $y$, and Corollary 2.5, it follows that $C$ is not a copula.

\section{Applications}

In this section we show how the new characterization given by Theorem 2.3 can be used for defining new families of copulas by generalizing existing methods.

\subsection{Patchwork of copulas}

In the recent literature, several researchers have considered constructions of bivariate copulas obtained by "patching" or "gluing" two or more copulas that take the same values on some subsets of the unit square, like diagonals and sections: see, for example, Nelsen (2006); De Baets and De Meyer (2007); Durante et al. (2007a); Siburg and Stoimenov (2008); Durante et al. (2009); Nelsen et al. (2008) and the references therein. Here we present a standard method for proving and generalizing results of this type.

Let us consider two closed subsets $\Omega$ and $\Omega^{\prime}$ of $\mathbb{I}^{2}$ such that $\Omega \cup \Omega^{\prime}=\mathbb{I}^{2}$ and $\Gamma=\Omega \cap \Omega^{\prime}$. Let $A: \Omega \rightarrow \mathbb{I}$ and $A^{\prime}: \Omega^{\prime} \rightarrow \mathbb{I}$ be continuous functions such that $A(x, y)=A^{\prime}(x, y)$ for every $(x, y) \in \Gamma$. Define the patchwork of $A$ and $A^{\prime}$ as the continuous function $C^{A, A^{\prime}}: \mathbb{I}^{2} \rightarrow \mathbb{I}$ given by

$$
C^{A, A^{\prime}}(x, y)= \begin{cases}A(x, y), & (x, y) \in \Omega, \\ A^{\prime}(x, y), & (x, y) \in \Omega^{\prime} \backslash \Gamma .\end{cases}
$$

Suppose that, for every point $x_{0} \in \mathbb{I} \backslash \mathscr{Z}$, where $\mathscr{Z}$ is a countable subset of $\mathbb{I}$, the intersection of the line $x=x_{0}$ with $\Gamma$ either is empty or consists of one point $\left(x_{0}, y_{0}\right)$ such that: 
(a) $\left\{x_{0}\right\} \times\left[0, y_{0}\right] \subseteq \Omega$ and $\left\{x_{0}\right\} \times\left[y_{0}, 1\right] \subseteq \Omega^{\prime}$,

(b) there exists $\varepsilon=\varepsilon_{\left(x_{0}, y_{0}\right)}>0$ for which the segment joining $\left(x_{0}, y_{0}\right)$ with $\left(x_{0}+\varepsilon, y_{0}\right)$ is entirely contained either in $\Omega$ or in $\Omega^{\prime}$,

(c) $D^{+} A_{y_{0}}\left(x_{0}\right) \leq D^{+} A_{y_{0}}^{\prime}\left(x_{0}\right)$.

Possible sets $\Gamma$ are shown in Figure 1. Note that the set $\mathscr{Z}$ could contain, for instance, all points $x_{0} \in \mathbb{I}$ such that the intersection of $\Gamma$ and the line $x=x_{0}$ consists of a segment, or the intersection is just given by the point $\left(x_{0}, y_{0}\right)$, but $\Gamma$ "oscillates" near $\left(x_{0}, y_{0}\right)$ and the condition (b) is not fulfilled.
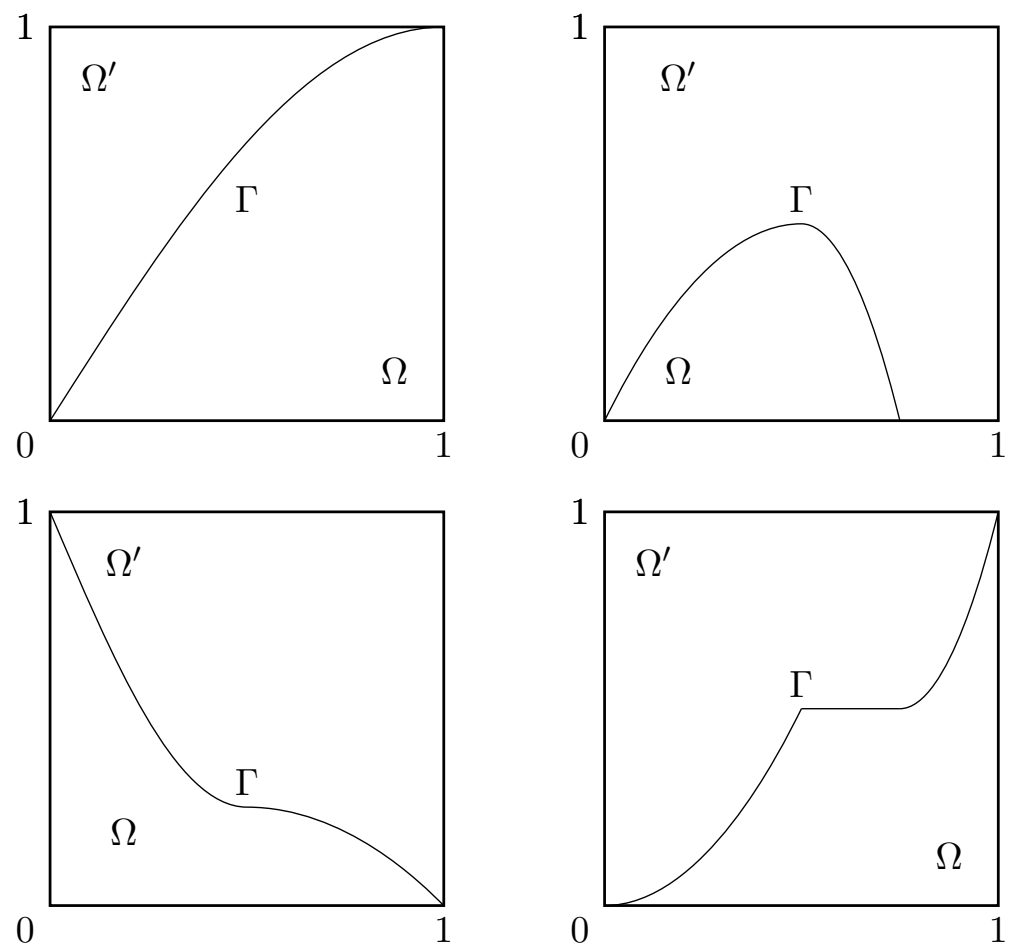

Figure 1: Examples of possible sets $\Omega, \Omega^{\prime}$ and $\Gamma$ of subsection 3.1

Proposition 3.1. Under the above assumptions, if $A$ and $A^{\prime}$ are copulas, then the function $C^{A, A^{\prime}}$ given by (3.1) is a copula.

Proof. In view of Theorem 2.3, we should just verify that $C^{A, A^{\prime}}$ satisfies conditions (C1), (C2), (C3') and (C4'). Since $A, A^{\prime}$ are copulas, the first three conditions are easily proved. Moreover, $A, A^{\prime}$ are 1 -Lipschitz and, hence, it is easy to show that $D^{+} C_{y}(x)$ is finite for every $x \in[0,1)$ and $y \in \mathbb{I}$. Now, for concluding the proof it suffices to show that, for all $x_{0} \in[0,1) \backslash \mathscr{Z}$, the function $\varphi_{x_{0}}: \mathbb{I} \rightarrow \mathbb{I}, \varphi_{x_{0}}(t)=D^{+} C_{t}\left(x_{0}\right)$ is increasing in $t$. The following cases should be considered. 
1. If the line $x=x_{0}$ does not intersect $\Gamma$, then $C$ coincides with either $A$ or $A^{\prime}$ on the segment $\left\{x_{0}\right\} \times \mathbb{I}$ and, in both cases, $\varphi_{x_{0}}$ is increasing.

2. If the line $x=x_{0}$ intersects $\Gamma$ at the point $\left(x_{0}, y_{0}\right)$, then:

- for every $y<y_{0}$ and for a sufficiently small $\varepsilon_{y}=\varepsilon>0, C=A$ on $\left[x_{0}, x_{0}+\epsilon\right] \times\{y\}$,

- for every $y^{\prime}>y_{0}$ and for a sufficiently small $\varepsilon_{y^{\prime}}=\varepsilon^{\prime}>0, C=A^{\prime}$ on $\left[x_{0}, x_{0}+\varepsilon\right] \times$ $\left\{y^{\prime}\right\}$.

Now, $\varphi_{x_{0}}(t)$ is increasing for every $t \in\left[0, y_{0}\right)$ and for every $t \in\left(y_{0}, 1\right]$. Condition (b) implies that $\varphi_{x_{0}}\left(y_{0}\right)$ equals $D^{+} A_{y_{0}}\left(x_{0}\right)$ or $D^{+} A_{y_{0}}^{\prime}\left(x_{0}\right)$. Therefore, due to condition (c), $\varphi_{x_{0}}(t)$ is increasing for every $t \in\left[0, y_{0}\right]$ and for every $t \in\left[y_{0}, 1\right]$, hence on $[0,1]$.

Thus, the assertion is true.

Note that the previous result includes as special case all the constructions related to the patchwork of copulas coinciding on the diagonal section, i.e. $\Gamma=\{(x, x) \mid x \in \mathbb{I}\}$ : see (Durante et al., 2007a; Nelsen et al., 2008). Moreover, it also applies to all the cases where two copulas $A$ and $A^{\prime}$ coincides on a given $\Gamma$ being the graph of a continuous and strictly increasing function of $\mathbb{I}$. For instance, $\Gamma$ could be the opposite diagonal section of $\mathbb{I}^{2}$ or an affine section (for such cases, see, for example, (Klement and Kolesárová, 2007; De Baets et al., 2009)).

\subsection{Copulas derived from suitable 2-increasing functions}

Here we describe a method for obtaining copulas starting with some arbitrary 2-increasing functions with suitable properties. First, we need the following auxiliary result.

Lemma 3.2. If the function $H: \mathbb{I}^{2} \rightarrow \mathbb{R}$ is 2-increasing, increasing in each variable and 1-Lipschitz, then, for every $i \in\{1,2,3,4\}$, the functions $C^{i}: \mathbb{I}^{2} \rightarrow \mathbb{I}$ defined by

$$
\begin{aligned}
& C^{1}(x, y)=\min (y, H(x, y)) \\
& C^{2}(x, y)=\min (x, H(x, y)) \\
& C^{3}(x, y)=\max (0, H(x, y)) \\
& C^{4}(x, y)=\max (x+y-1, H(x, y))
\end{aligned}
$$

are also 2-increasing, increasing in each variable and 1-Lipschitz.

Proof. It can be easily verified that, for every $i \in\{1,2,3,4\}, C^{i}$ is increasing in each variable and 1-Lipschitz. Then, it remains to check only the 2 -increasing property of $C^{i}$. Since all the proofs can be similarly done, we just show here that $C^{1}=C$ is 2-increasing.

First, observe that, for all $y \in \mathbb{I}$ and all $x \in[0,1), 0 \leq D^{+} H_{y}(x) \leq 1$, and, for every $x \in \mathbb{I}$, the functions $\Psi_{1}(y)=H(x, y)$ and $\Psi_{2}(y)=y-H(x, y)$ are increasing on $\mathbb{I}$ (note that $\Psi_{2}$ is increasing because of $H$ is 1-Lipschitz). 
Consider the following subsets of $\mathbb{I}^{2}$ :

$$
\Omega=\left\{(x, y) \in \mathbb{I}^{2} \mid H(x, y)<y\right\}, \quad \Omega^{\prime}=\mathbb{I}^{2} \backslash \Omega
$$

Suppose that $\Omega$ and $\Omega^{\prime}$ are nonempty (otherwise, the proof is trivial). Therefore, we have

$$
C(x, y)= \begin{cases}H(x, y), & (x, y) \in \Omega \\ y, & (x, y) \in \Omega^{\prime}\end{cases}
$$

Let $x_{0} \in[0,1)$. In order to prove that $C$ is 2 -increasing, it suffices to show that the function $\varphi_{x_{0}}: \mathbb{I} \rightarrow \mathbb{R}, \varphi_{x_{0}}(t)=D^{+} C_{t}\left(x_{0}\right)$ is increasing in $t \in \mathbb{I}$. The proof will then follow by using Lemma 2.2.

Let $y_{0} \in \mathbb{I}$ such that $H\left(x_{0}, y_{0}\right)=y_{0}$ (when such a point does not exist, the proof is immediate). Since $\Psi_{2}$ is increasing, we have that $C\left(x_{0}, y\right)=H\left(x_{0}, y\right)$ for every $y>y_{0}$ and, analogously, $C\left(x_{0}, y\right)=y$ for every $y<y_{0}$. Now, since $H$ is increasing in first variable, we get that, for sufficiently small $\varepsilon>0$, if $H\left(x_{0}, y\right) \geq y$, then $H\left(x_{0}+\varepsilon, y\right) \geq y$ as well. In this case $C\left(x_{0}+\varepsilon, y\right)=C\left(x_{0}, y\right)=y$ and $D^{+} C_{y}\left(x_{0}\right)=0$. Thus, $\varphi_{x_{0}}(t)=0$ for every $t \in\left[0, y_{0}\right]$. Moreover, since $H$ is continuous, we get that, for sufficiently small $\varepsilon>0$, if $H\left(x_{0}, y\right)<y$ then $H\left(x_{0}+\varepsilon, y\right)<y$ as well. In this case $C\left(x_{0}+\varepsilon, y\right)=H\left(x_{0}+\varepsilon, y\right), C\left(x_{0}, y\right)=H\left(x_{0}, y\right)$ and $D^{+} C_{y}\left(x_{0}\right)=D^{+} H_{y}(x)$. Therefore, $\varphi_{x_{0}}(t)=D^{+} H_{t}(x)$ for every $t \in\left[x_{0}, 1\right]$. Since $H$ is 2 -increasing, it follows that $\varphi_{x_{0}}$ is increasing on $\mathbb{I}$.

Proposition 3.3. If the function $H: \mathbb{I}^{2} \rightarrow \mathbb{R}$ is 2 -increasing, increasing in each variable and 1-Lipschitz, then the function $C: \mathbb{I}^{2} \rightarrow \mathbb{I}$ defined by

$$
C(x, y)=\max (\min (x, y, H(x, y)), 0, x+y-1)
$$

is a copula.

Proof. It is easy to show that the function $C$ given by (3.6) satisfies (C1), (C2) and (C3'). In order to prove that $C$ is 2 -increasing it is enough to apply Lemma 3.2, since $C$ can be obtained as a suitable combination of the functions $C^{i}$ defined by (3.2)-(3.5).

Intuitively speaking, copulas of type (3.6) can be obtained by forcing a suitable function $H$, which satisfies all the properties of a copula apart from the boundaries conditions (C1) and $(\mathrm{C} 2)$, to be bounded from above by the copula $M(x, y)=\min (x, y)$, and from below by the copula $W(x, y)=\max (x+y-1,0)$. This construction is, hence, trivial in the case $H$ is a copula and, hence, $C=H$. In all other cases, it may give several other copulas. When $H(x, y) \geq W(x, y)$ on $\mathbb{I}^{2}$, it reduces to the construction described by Durante et al. (2007b), which includes the diagonal copulas given by Fredricks and Nelsen (1997).

Here we show another possible use of copulas of type (3.6). Jaworski (2004) has shown how to construct copulas with a given leading part that is homogeneous of degree 1 , i.e. 
copulas $C$ such that, for every $(x, y) \in \mathbb{R}_{+}^{2}=[0,+\infty)^{2}$, the ray-like limits

$$
\lim _{t \rightarrow 0^{+}} \frac{C(t x, t y)}{t}
$$

exist and are equal to a prescribed homogeneous function $Q: \mathbb{R}_{+}^{2} \rightarrow \mathbb{R}$ of degree 1 . It can be verified that such function should be non-negative, grounded, 2-increasing and bounded by the minimum, i.e. $Q(x, y) \leq \min (x, y)$ for every $(x, y) \in \mathbb{R}_{+}^{2}$. Such constructions have been used for the risk estimation of bivariate extreme events (compare also with (Jaworski, 2006; Joe et al., 2008; Joe and Li, 2009)). Below we extend this result to copulas with homogeneous leading part of degree $d>1$.

Proposition 3.4. Let $Q: \mathbb{R}_{+}^{2} \rightarrow \mathbb{R}_{+}$be a 2-increasing function, which is homogeneous of degree $d, d>1$, and Lipschitz on $\mathbb{I}^{2}$. Then, there exists a copula $C$ such that, for all $(x, y) \in$ $(0,+\infty)^{2}$

$$
\lim _{t \rightarrow 0^{+}} \frac{C(t x, t y)}{t^{d}}=Q(x, y) .
$$

Proof. Let $\Lambda$ be the Lipschitz constant of the restriction of $Q$ to $\mathbb{I}^{2}$, i.e.

$$
\forall x_{1}, x_{2}, y_{1}, y_{2} \in \mathbb{I} \quad\left|Q\left(x_{1}, y_{1}\right)-Q\left(x_{2}, y_{2}\right)\right| \leq \Lambda\left(\left|x_{1}-x_{2}\right|+\left|y_{1}-y_{2}\right|\right) .
$$

If $\Lambda \leq 1$, then we consider the function $C: \mathbb{I}^{2} \rightarrow \mathbb{R}_{+}$defined by

$$
C(x, y)=\max (\min (x, y, Q(x, y)), x+y-1) .
$$

Since the margins $x \mapsto Q(x, 0)=x^{d} Q(1,0)$ and $y \mapsto Q(0, y)=y^{d} Q(0,1)$ are increasing and $Q$ is 2-increasing, it can be proved that $Q$ is increasing in each argument, when the other is held fixed. Thus, from Proposition 3.3 it follows that $C$ is a copula. Since $d>1$, we get that, for all $(x, y) \in(0,+\infty)^{2}$ and for any sufficiently small $t>0$,

$$
\begin{aligned}
C(t x, t y) & =\max (\min (t x, t y, Q(t x, t y)), t x+t y-1) \\
& =\min \left(t x, t y, t^{d} Q(x, y)\right)=t^{d} Q(x, y)
\end{aligned}
$$

Hence

$$
\lim _{t \rightarrow 0^{+}} \frac{C(t x, t y)}{t^{d}}=\lim _{t \rightarrow 0^{+}} \frac{t^{d} Q(x, y)}{t^{d}}=Q(x, y) .
$$

If $\Lambda>1$, then we consider the function $C^{*}: \mathbb{I}^{2} \rightarrow \mathbb{R}_{+}$defined by

$$
C^{*}(x, y)=\max \left(\min \left(x, y, \Lambda^{-1} Q(x, y)\right), x+y-1\right) .
$$

The same arguments used as above imply that $C^{*}$ is a copula. Consider, now, the copula $C$ that is an ordinal sum of $C^{*}$ and the copula $M(x, y)=\min (x, y)$ with respect to the intervals $(0, a)$ and $(a, 1)$, where $a^{1-d}=\Lambda$, i.e.

$$
C(x, y)= \begin{cases}a C^{*}\left(\frac{x}{a}, \frac{y}{a}\right), & (x, y) \in[0, a]^{2} \\ \min (x, y), & \text { otherwise }\end{cases}
$$


So, for every $(x, y) \in[0, a]^{2}$, easy calculations show that

$$
C(x, y)=\max (\min (x, y, Q(x, y)), x+y-a)
$$

and, in the same way as above, we get that, for all $(x, y) \in(0,+\infty)^{2}$ and for any sufficiently small $t>0$,

$$
\lim _{t \rightarrow 0^{+}} \frac{C(t x, t y)}{t^{d}}=\lim _{t \rightarrow 0^{+}} \frac{t^{d} Q(x, y)}{t^{d}}=Q(x, y),
$$

which is the desired assertion.

\subsection{Modification of an absolutely continuous copula}

A well-known class of copulas is the Farlie-Gumbel-Morgenstern family (FGM, for short), whose members are given, for every $\alpha \in[-1,1]$, by

$$
C_{\alpha}(x, y)=x y+\alpha x y(1-x)(1-y)
$$

A complete survey on FGM models of dependence is given by Drouet-Mari and Kotz (2001).

From a construction viewpoint, FGM copulas are obtained by means of a suitable modification of the copula $\Pi(x, y)=x y$, expressing the independence among random variables, with some suitable functions $f(x)$ and $g(y)$ (compare with (Rodríguez-Lallena and Úbeda-Flores, 2004; Cuadras, 2009)), or, in other terms, by means of some modification of the density of the copula $\Pi$ (compare with (Rüschendorf, 1985)). Here we propose a method for defining new copulas by modifying an absolutely continuous one (similar ideas have been considered in Kim and Sungur (2004)).

Proposition 3.5. Let $C$ be an absolutely continuous copula with a continuous density $c$ and let $f, g: \mathbb{I} \rightarrow \mathbb{R}$ be continuous functions such that

$$
f(0)=f(1)=g(0)=g(1)=0 .
$$

Then the following conditions are equivalent:

(i) the function $H: \mathbb{I}^{2} \rightarrow \mathbb{R}$ given by $H(x, y)=C(x, y)+f(x) g(y)$ is a copula.

(ii) For every $x, y \in[0,1)$,

$$
\begin{aligned}
c(x, y) \geq-\min \left(D^{+} f(x) D^{+} g(y), D_{+} f(x) D^{+} g(y),\right. & \\
& \left.D^{+} f(x) D_{+} g(y), D_{+} f(x) D_{+} g(y)\right) .
\end{aligned}
$$

Proof. First, note that, since

$$
C(x, y)=\int_{0}^{x} \int_{0}^{y} c(s, t) d t d s
$$


we obtain that

$$
D^{+} C_{y}(x)=D_{+} C_{y}(x)=\frac{\partial C(x, y)}{\partial x}=\int_{0}^{y} c(x, t) d t
$$

is continuous in both $x$ and $y$. Moreover, for a fixed $x \in \mathbb{I}$, we get

$$
D^{+}\left(\frac{\partial C(x, y)}{\partial x}\right)=D_{+}\left(\frac{\partial C(x, y)}{\partial x}\right)=\frac{\partial^{2} C(x, y)}{\partial x \partial y}=c(x, y) .
$$

Now, let us introduce two auxiliary functions, depending on the parameter $x \in[0,1)$, given by

$$
\begin{aligned}
& \Psi_{x}(y)=\mathbf{1}_{g(y) \geq 0} D^{+} H_{y}(x)+\mathbf{1}_{g(y)<0} D_{+} H_{y}(x), \\
& \Phi_{x}(y)=\mathbf{1}_{g(y) \geq 0} D_{+} H_{y}(x)+\mathbf{1}_{g(y)<0} D^{+} H_{y}(x) .
\end{aligned}
$$

Since, for a constant $a \in \mathbb{R}$, we have

$$
D^{+}(a f(x))= \begin{cases}a D^{+} f(x), & a \geq 0, \\ a D_{+} f(x), & a<0,\end{cases}
$$

due to differentiability of $C_{y}$, it holds that

$$
\begin{aligned}
\Psi_{x}(y) & =\mathbf{1}_{g(y) \geq 0}\left(\frac{\partial C(x, y)}{\partial x}+g(y) D^{+} f(x)\right)+\mathbf{1}_{g(y)<0}\left(\frac{\partial C(x, y)}{\partial x}+g(y) D^{+} f(x)\right) \\
& =\frac{\partial C(x, y)}{\partial x}+g(y) D^{+} f(x)
\end{aligned}
$$

and, analogously,

$$
\Phi_{x}(y)=\frac{\partial C(x, y)}{\partial x}+g(y) D_{+} f(x)
$$

In the same way, we obtain

$$
\begin{aligned}
& \mathbf{1}_{D^{+} f(x) \geq 0} D^{+} \Psi_{x}(y)+\mathbf{1}_{D^{+} f(x)<0} D_{+} \Psi_{x}(y)=c(x, y)+D^{+} f(x) D^{+} g(y), \\
& \mathbf{1}_{D^{+} f(x) \geq 0} D_{+} \Psi_{x}(y)+\mathbf{1}_{D^{+} f(x)<0} D^{+} \Psi_{x}(y)=c(x, y)+D^{+} f(x) D_{+} g(y), \\
& \mathbf{1}_{D_{+} f(x) \geq 0} D^{+} \Phi_{x}(y)+\mathbf{1}_{D_{+} f(x)<0} D_{+} \Phi_{x}(y)=c(x, y)+D_{+} f(x) D^{+} g(y), \\
& \mathbf{1}_{D_{+} f(x) \geq 0} D_{+} \Phi_{x}(y)+\mathbf{1}_{D_{+} f(x)<0} D^{+} \Phi_{x}(y)=c(x, y)+D_{+} f(x) D_{+} g(y) .
\end{aligned}
$$

Now, suppose that $H: \mathbb{I}^{2} \rightarrow \mathbb{R}$ given by $H(x, y)=C(x, y)+f(x) g(y)$ is a copula. Then both $D^{+} H_{y}(x)$ and $D_{+} H_{y}(x)$ are increasing in $y$, and, hence, so are $\Psi_{x}(y)$ and $\Phi_{x}(y)$. Indeed $\Psi_{x}(y)$ and $\Phi_{x}(y)$ are continuous and equal to $D^{+} H_{y}(x)$ or $D_{+} H_{y}(x)$ in intervals where the sign of $g$ is constant. Therefore the Dini derivatives of $\Psi_{x}(y)$ and $\Phi_{x}(y)$ are nonnegative and

$$
c(x, y)+\min \left(D^{+} f(x) D^{+} g(y), D_{+} f(x) D^{+} g(y), D^{+} f(x) D_{+} g(y), D_{+} f(x) D_{+} g(y)\right) \geq 0 .
$$

Conversely, suppose that Eq. (3.8) is valid. Then the Dini derivatives of $\Psi_{x}(y)$ and $\Phi_{x}(y)$ are nonnegative. Hence both $\Psi_{x}(y)$ and $\Phi_{x}(y)$ are increasing and $D^{+} H_{y}(x)$ is increasing in $y$ as well. It is also finite, since $H_{0}(x)=H(x, 0)=C(x, 0)=0$ and $H_{1}(x)=H(1, x)=C(x, 1)=$ $x$, which imply that $D^{+} H_{0}(x)=0$ and $D^{+} H_{1}(x)=1$. Therefore, due to Theorem $2.3, H$ is a copula. 
When $C=\Pi$, the previous characterization should be compared with (Rodríguez-Lallena and Úbeda-Flores, 2004, Theorem 2.3).

\section{Acknowledgments}

We would like to express our gratitude to an anonymous Reviewer for her/his careful reading and useful comments that improved the presentation of the manuscript.

This work was supported by the project Copula Theory and its Applications, bilateral cooperation Austria-Poland (WTZ PL 03/2008). This work was also partially conducted during the Special Semester on Stochastic with Emphasis on Finance, September 3 - December 5, 2008, organized by RICAM (Johann Radon Institute for Computational and Applied Mathematics, Austrian Academy of Sciences), Linz, Austria.

\section{References}

Alsina, C., Nelsen, R. B., and Schweizer, B. (1993). On the characterization of a class of binary operations on distribution functions. Statist. Probab. Lett., 17(2):85-89.

Cuadras, C. M. (2009). Constructing copula functions with weighted geometric means. J. Statist. Plan. Infer. in press.

De Baets, B. and De Meyer, H. (2007). Orthogonal grid constructions of copulas. IEEE Trans. Fuzzy Systems, 15(6):1053-1062.

De Baets, B., De Meyer, H., and Úbeda-Flores, M. (2009). Opposite diagonal sections of quasi-copulas and copulas. Internat. J. Uncertain. Fuzziness Knowledge-Based Systems. In press.

Drouet-Mari, D. and Kotz, S. (2001). Correlation and dependence. Imperial College Press, London.

Durante, F., Kolesárová, A., Mesiar, R., and Sempi, C. (2007a). Copulas with given diagonal sections: novel constructions and applications. Internat. J. Uncertain. Fuzziness Knowledge-Based Systems, 15(4):397-410.

Durante, F., Mesiar, R., Papini, P.-L., and Sempi, C. (2007b). 2-increasing binary aggregation operators. Inform. Sci., 177(1):111-129.

Durante, F., Saminger-Platz, S., and Sarkoci, P. (2009). Rectangular patchwork for bivariate copulas and tail dependence. Comm. Statist. Theory Methods. In press.

Embrechts, P. (2009). Copulas: a personal view. Journal of Risk and Insurance. in press.

Fredricks, G. A. and Nelsen, R. B. (1997). Copulas constructed from diagonal sections. In Beneš, V. and Štěpán, J., editors, Distributions with given marginals and moment problems (Prague, 1996), pages 129-136. Kluwer Acad. Publ., Dordrecht.

Genest, C., Quesada-Molina, J. J., Rodríguez-Lallena, J. A., and Sempi, C. (1999). A characterization of quasi-copulas. J. Multivariate Anal., 69(2):193-205. 
Jaworski, P. (2004). On uniform tail expansions of bivariate copulas. Appl. Math. (Warsaw), 31(4):397415.

Jaworski, P. (2006). On value at risk for foreign exchange rates - the copula approach. Acta Phys. Polon. B, 37(11):3005-3015.

Joe, H. (1997). Multivariate models and dependence concepts, volume 73 of Monographs on Statistics and Applied Probability. Chapman \& Hall, London.

Joe, H. and Li, H. (2009). Tail risk of multivariate regular variation. Technical report.

Joe, H., Li, H., and Nikoloulopoulos, A. (2008). Tail dependence functions and vine copulas. Technical Report 2008-3, Department of Mathematics - Washington State University.

Kim, J.-M. and Sungur, E. A. (2004). New class of bivariate copulas. In Proceedings for the Spring Conference of Korean Statistical Society, pages 207-212.

Klement, E. P. and Kolesárová, A. (2007). Intervals of 1-Lipschitz aggregation operators, quasi-copulas, and copulas with given affine section. Monatsh. Math., 152(2):151-167.

Łojasiewicz, S. (1988). An introduction to the theory of real functions. A Wiley-Interscience Publication. John Wiley \& Sons Ltd., Chichester, third edition.

McNeil, A. J., Frey, R., and Embrechts, P. (2005). Quantitative risk management. Concepts, techniques and tools. Princeton Series in Finance. Princeton University Press, Princeton, NJ.

Nelsen, R. B. (2006). An introduction to copulas. Springer Series in Statistics. Springer, New York, second edition.

Nelsen, R. B., Quesada-Molina, J. J., Rodríguez-Lallena, J. A., and Úbeda-Flores, M. (2008). On the construction of copulas and quasi-copulas with given diagonal sections. Insurance Math. Econom., $42(2): 473-483$.

Rodríguez-Lallena, J. A. and Úbeda-Flores, M. (2004). A new class of bivariate copulas. Statist. Probab. Lett., 66(3):315-325.

Rüschendorf, L. (1985). Construction of multivariate distributions with given marginals. Ann. Inst. Statist. Math., 37(2):225-233.

Salvadori, G., De Michele, C., Kottegoda, N. T., and Rosso, R. (2007). Extremes in Nature. An Approach Using Copulas, volume 56 of Water Science and Technology Library. Springer, Dordrecht $(\mathrm{NL})$.

Schweizer, B. and Sklar, A. (2006). Probabilistic Metric Spaces. Dover Publications, Mineola, N. Y.

Siburg, K. F. and Stoimenov, P. A. (2008). Gluing copulas. Comm. Statist. Theory Methods, 37(19):3124-3134. 